\title{
Surges and Si IV Bursts in the Solar Atmosphere: Understanding IRIS and SST Observations through RMHD Experiments
}

\author{
D. Nóbrega-Siverio ${ }^{1,2}$ (10), J. Martínez-Sykora ${ }^{3,4}$ (i) , F. Moreno-Insertis ${ }^{1,2}$, and L. Rouppe van der Voort ${ }^{5}$ (1) \\ ${ }^{1}$ Instituto de Astrofisica de Canarias, Via Lactea, s/n, E-38205 La Laguna (Tenerife), Spain; dnobrega@iac.es \\ ${ }^{2}$ Department of Astrophysics, Universidad de La Laguna, E-38200 La Laguna (Tenerife), Spain; fmi@iac.es \\ ${ }^{3}$ Lockheed Martin Solar and Astrophysics Laboratory, Palo Alto, CA 94304, USA; juanms@1msal.com \\ ${ }^{4}$ Bay Area Environmental Research Institute, Petaluma, CA 94952, USA \\ ${ }^{5}$ Institute of Theoretical Astrophysics, University of Oslo, P.O. Box 1029 Blindern, NO-0315 Oslo, Norway; rouppe @ astro.uio.no \\ Received 2017 August 18; revised 2017 October 20; accepted 2017 October 20; published 2017 November 28
}

\begin{abstract}
Surges often appear as a result of the emergence of magnetized plasma from the solar interior. Traditionally, they are observed in chromospheric lines such as $\mathrm{H} \alpha 6563 \AA$ and Ca II $8542 \AA$. However, whether there is a response to the surge appearance and evolution in the Si IV lines or, in fact, in many other transition region lines has not been studied. In this paper, we analyze a simultaneous episode of an $\mathrm{H} \alpha$ surge and a Si IV burst that occurred on 2016 September 03 in active region AR 12585. To that end, we use coordinated observations from the Interface Region Imaging Spectrograph and the Swedish 1-m Solar Telescope. For the first time, we report emission of Si IV within the surge, finding profiles that are brighter and broader than the average. Furthermore, the brightest Si IV patches within the domain of the surge are located mainly near its footpoints. To understand the relation between the surges and the emission in transition region lines like Si IV, we have carried out 2.5D radiative MHD (RMHD) experiments of magnetic flux emergence episodes using the Bifrost code and including the nonequilibrium ionization of silicon. Through spectral synthesis, we explain several features of the observations. We show that the presence of Si IV emission patches within the surge, their location near the surge footpoints and various observed spectral features are a natural consequence of the emergence of magnetized plasma from the interior to the atmosphere and the ensuing reconnection processes.
\end{abstract}

Key words: line: profiles - magnetohydrodynamics (MHD) - methods: observational - methods: numerical - Sun: chromosphere - Sun: transition region

Supporting material: animations

\section{Introduction}

Surges are a good example of the complexity of chromospheric ejections. In $\mathrm{H} \alpha 6563 \AA$, surges are seen as darkenings in the blue/red wings of the line with line-of-sight (LOS) apparent velocities of a few to several tens of $\mathrm{km} \mathrm{s}^{-1}$ on areas with projected lenghts of 10-50 Mm (Kirshner \& Noyes 1971; Roy 1973; Cao et al. 1980; Schmieder et al. 1984; Chae et al. 1999; Guglielmino et al. 2010, among others). They can be recurrent (Schmieder et al. 1995; Gaizauskas 1996; Jiang et al. 2007; Uddin et al. 2012) and have apparent rotational and helical motions (Gu et al. 1994; Canfield et al. 1996; Jibben \& Canfield 2004; Bong et al. 2014). Recent observations show that the surges consist of small-scale thread-like structures (Nelson \& Doyle 2013; Li et al. 2016) and that they appear to be related to shocks (Yang et al. 2014) and Kelvin-Helmholtz instabilities (Zhelyazkov et al. 2015). The surges have also been detected in other chromospheric lines, such as the Ca II $8542 \AA$ infrared triplet (Yang et al. 2013; Kim et al. 2015), He I $10830 \AA$ (Vargas Domínguez et al. 2014), H $\beta 4861 \AA$ (Zhang et al. 2000; Liu \& Kurokawa 2004), and also in Ca II H \& K (Rust 1976; Nishizuka et al. 2008; Liu et al. 2009).

Surges normally appear to be related with emerging flux regions (EFR, Kurokawa et al. 2007). This is especially evident in active regions (ARs), where there are many observations of such cool ejections (e.g., Brooks et al. 2007; Madjarska et al. 2009; Wang et al. 2014). Furthermore, surges are also associated with other phenomena such as light bridges (Asai et al. 2001; Shimizu et al. 2009; Robustini et al. 2016) and explosive events (EEs), EUV or X-ray ejections (see, e.g., Schmahl 1981; Canfield et al. 1996; Chen et al. 2008; Madjarska et al. 2009; Zhang \& Ji 2014). It has also been suggested that surges can be related to Ellerman bombs (EBs; Watanabe et al. 2011; Vissers et al. 2013; Yang et al. 2013); nevertheless, this relation does not seem to be common (Rutten et al. 2013).

On the other hand, observations carried out with the Interface Region Imaging Spectrograph (IRIS) satellite (De Pontieu et al. 2014) have provided a new perspective on transient phenomena in the chromosphere and transition region. In particular, the connection between UV bursts observed with IRIS (also referred to as IRIS bursts or IRIS bombs; Peter et al. 2014) and previously known phenomena has become an active area of research (see, e.g., Judge 2015; Vissers et al. 2015; Tian et al. 2016; Grubecka et al. 2016, among others). In this context, recent papers report on the coexistence of surges with bursts in Si IV (Kim et al. 2015, Huang et al. 2017 and M. S. Madjarska et al. 2017, in preparation); nevertheless, the focus of those papers was mainly on the bursts, without presenting an in-depth analysis of the associated surges.

The aim of this paper is to explore the response of transition region lines during surge formation and to characterize and explain the corresponding spectral profiles. Furthermore, we want to provide theoretical support for the relation between $\mathrm{H} \alpha$ surges and other phenomena such as Si IV bursts and EBs. To that end, we use coordinated observations from space and the ground with IRIS and the Swedish 1-m Solar Telescope (SST; Scharmer et al. 2003). Those instruments provide high-resolution observations 
that cover chromospheric and transition region lines, which are essential for this study. Theoretical support is given by $2.5 \mathrm{D}$ numerical experiments similar to the one discussed in detail by Nóbrega-Siverio et al. (2016, hereafter NS2016), but extending the Bifrost code capabilities (Gudiksen et al. 2011) by including a module developed by Olluri et al. (2013b) that calculates the ionization state of the Si IV in nonequilibrium (NEQ).

The layout of the paper is as follows. Section 2 describes the IRIS and SST coordinated observations, and briefly the numerical models. In Section 3, we analyze the observations showing mainly Si IV profiles, Doppler shifts, and line widths in the region of interest. In Section 4, we study the synthetic spectral profiles obtained from the numerical experiments, comparing them with the observations. Finally, Section 5 contains the conclusions and a summary.

\section{Observations and Numerical Model}

\subsection{Coordinated Observations}

For this paper, we have used coordinated observations of IRIS and SST obtained on 2016 September 03 from 07:49:21 to 10:03:37 UT centered on active region AR 12585 at heliocentric coordinates $(x, y)=\left(-561^{\prime \prime}, 44^{\prime \prime}\right)$ and observing angle $\mu=0.8$.

1. The IRIS data set corresponds to the observation program OBSID: 3625503135. Those observations cover a maximum field of view (FOV) of $65^{\prime \prime} \times 60^{\prime \prime}$. The spectral information is obtained through a medium-dense raster that covers $60^{\prime \prime}$ in the $y$ direction and scans 5.'28 in the $x$ direction in 16 steps of 0 ." 33 separation for each step, with a raster cadence of $20 \mathrm{~s}$. The exposure time is $0.5 \mathrm{~s}$, the spatial scale is 0 ". 166 pixel $^{-1}$, and the spectral sampling of the FUV spectra has been binned four times in the camera from 2.7 to $11 \mathrm{~km} \mathrm{~s}^{-1}$ to increase the signal level. Concerning the slit-jaw images (SJI), C II 1330, Si IV 1400, and Mg II k $2796 \AA$ were taken with a cadence of $10 \mathrm{~s}$ obtaining a total of 800 images in each passband. The data have been calibrated for dark current, flat-field, and geometric correction. We analyze level 2 data (De Pontieu et al. 2014) produced by SolarSoft's iris_prep.pro routine version 1.83, which includes an updated version of the wavelength calibration (level2 version L12-2017-04-23). When studying IRIS FUV spectra profiles in the following, we shall consider temporal and spatial evolution of features like intensities, relative velocities, line widths, and asymmetries. On the other hand, the absolute wavelength calibration of these observations is not good enough to allow for a determination with accuracy better than $20-25 \mathrm{~km} \mathrm{~s}^{-1}$ of the global Doppler shift. This is due to the lack of photospheric lines in this data set as a result of the short exposures.

2. The SST data set contains Ca II $8542 \AA$ and H $\alpha 6563 \AA$ spectral scans obtained with the SST/CRISP Fabry-Pérot Interferometer (Scharmer 2006). The CRISP scans have a temporal cadence of $20 \mathrm{~s}$, a spatial sampling of 0 !" 057 per pixel and an FOV of $\sim 58^{\prime \prime} \times 58^{\prime \prime}$. The diffraction limit of the SST at $6563 \AA$ is 0 ." 14 . H $\alpha$ was sampled at 15 line positions from $-1500 \mathrm{~m} \AA$ to $+1500 \mathrm{m \AA}$ (FWHM transmission profile: $66 \mathrm{m \AA}$ ), and Ca II 8542 was sampled in the spectropolarimetric mode at 21 spectral positions between $-1750 \mathrm{~m} \AA$ and $+1750 \mathrm{~m} \AA$ offset from the line core. The wings were sparsely sampled and the line core region was sampled with equidistant steps of $70 \mathrm{~m} \AA$ between $\pm 455 \mathrm{~m} \AA$ (FWHM transmission profile: $107 \mathrm{~m} \AA$ ). Taking into account the magnetic sensitivity and the wide range in formation height of the Ca II 8542 line (see, e.g., de la Cruz Rodriguez 2010), we construct photospheric polarization maps by adding the Stokes $V$ maps of the three outer spectral positions in both wings, preserving the sign of magnetic polarity by effectively subtracting the sum of the three red wing maps from the sum of the three blue wing maps. While the Ca II 8542 line core originates from the chromosphere, the far wings originate from the photosphere, so these accumulated polarization maps serve as a proxy for the photospheric magnetic field (de la Cruz Rodríguez et al. 2012; Ortiz et al. 2014). The CRISP data were processed following the CRISPRED data reduction pipeline (de la Cruz Rodríguez et al. 2015), which includes Multi-Object Multi-Frame Blind Deconvolution image restoration (van Noort et al. 2005). The alignment with the IRIS data was done by scaling down the CRISP data to the IRIS pixelscale and through cross-correlation of the Ca II $8542 \AA$ wing images with the IRIS SJI 2796 channel. This alignment has been shown to be accurate down to the level of the IRIS pixel (0!'17, Rouppe van der Voort et al. 2015).

Figure 1 shows a fraction of the FOV of the region observed by IRIS and SST at $\sim 99: 39$ UT. Panel (A) shows a polarization map of the Ca II $8542 \AA$ wings that serves as a proxy of the photospheric magnetic field in active region AR 12585. Panel (B) contains the IRIS SJI 1400; and panel (C) is the SST image in the blue wing of $\mathrm{H} \alpha$ at $-46 \mathrm{~km} \mathrm{~s}^{-1}$ (an accompanying movie of the figure is provided in the online version). The solid rectangle in all three panels identifies the area of interest where the events originate. Additionally, part of that region is also covered by the IRIS raster (see the area within the vertical dashed lines in the figure), so we have detailed Si IV spectral information from part of the surge and burst to compare with the synthetic spectra of the numerical experiment. In panel (B), the arrows mark two regions, R1 and R2, that correspond to a surge and a burst, respectively, and which are explained in detail in Section 3. At the time of the figure, approximately at 09:39 UT, two opposite polarities are colliding within the solid rectangle (see panel (A)), while in panel (B) a Si IV burst (green contour) is visible around $(x, y)=\left(-557^{\prime \prime}, 51^{\prime \prime}\right)$. Co-spatial with the upper part of the bright region in the SJI 1400 at $(x, y)=\left(-558^{\prime \prime}, 51^{\prime \prime}\right)$, we identify an intense brightening in the $\mathrm{H} \alpha$ wing in panel (C), which is highlighted with a red contour within the burst. The $\mathrm{H} \alpha$ spectral profile reveals that this is an EB (see Section 3). The blue contours in the three panels mark out dark structures visible in panel (C). Some of those dark structures correspond to $\mathrm{H} \alpha$ surges; in particular, the relevant one for this paper is marked by the R1 arrow and appears during the Si IV burst (R2). Following the darkenings in the SST images, it seems that this surge is ejected leftwards of $(x, y) \sim\left(-559^{\prime \prime}, 49^{\prime \prime}\right)$; we will refer to that location as the footpoint of the surge in the following.

\subsection{The Numerical Experiment}

In order to understand the physics of the surges and related phenomena, it is necessary to provide theoretical support to the 
A) Stokes V Ca II 8542

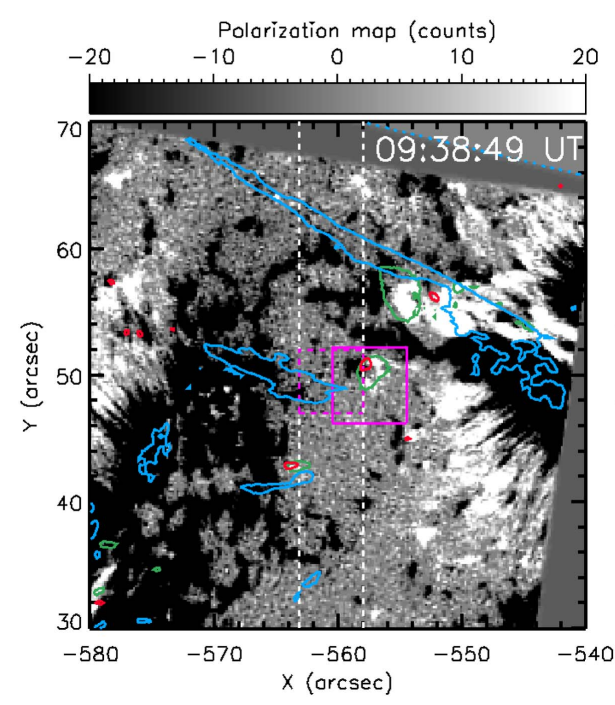

B) SJl 1400

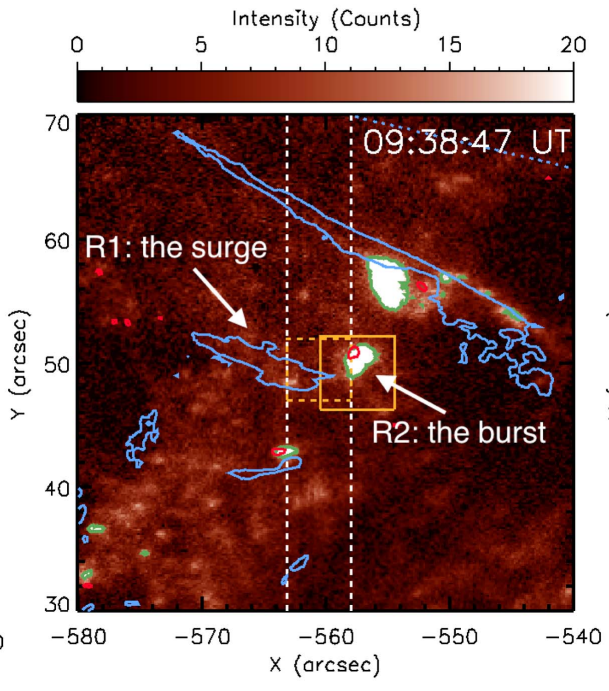

C) Halpha blue wing

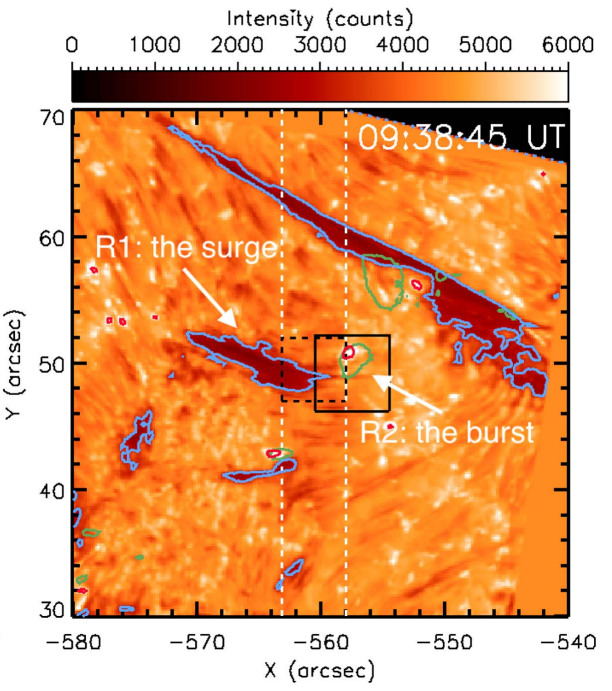

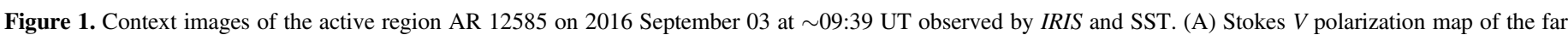

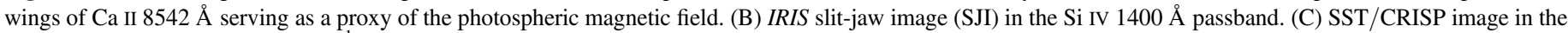

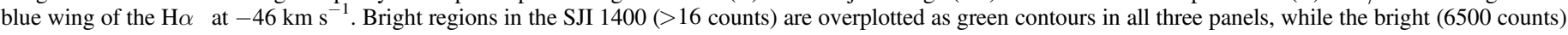

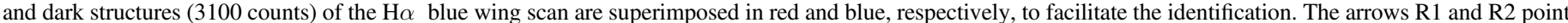

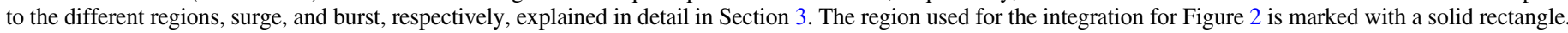

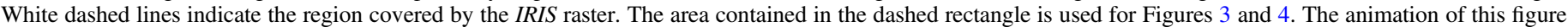
shows the time evolution of the three panels from $\sim 08: 56$ UT to the end of the coordinated observations at $\sim 10: 03$ UT.

(An animation of this figure is available.)

observations. To that end, we have run two 2.5D numerical experiments in which a surge results from magnetic flux emergence and where thermal conduction and radiative transfer are treated in a self-consistent manner.

1. Concerning the code, the present experiments have been performed with the 3D RMHD Bifrost code (Gudiksen et al. 2011; Carlsson \& Leenaarts 2012; Hayek et al. 2010). In addition, we have enabled a module to calculate the nonequilibrium (NEQ) ionization for silicon developed by Olluri et al. (2013b). Thus, we are able to compute the emissivity and then the synthetic spectra under optically thin conditions, as in the papers by Olluri et al. (2015) and Martínez-Sykora et al. (2016), for different orientations of the $\operatorname{LOS}\left(-15^{\circ}, 0^{\circ}\right.$, and $\left.15^{\circ}\right)$ with respect to the vertical direction $z$.

2. Regarding the numerical setup, the two runs are similar to the one in the NS2016 paper. The main differences are as follows: (1) we have kept the resolution of NS2016 but increased the physical domain, namely, $0.0 \mathrm{Mm}$ $\leqslant x \leqslant 32.0 \mathrm{Mm}$ and $-2.6 \mathrm{Mm} \leqslant z \leqslant 30.0 \mathrm{Mm}$, where $z=0 \mathrm{Mm}$ corresponds to the solar surface; (2) one of the current experiments has a vertical coronal magnetic field while the other is slanted; and (3) the initial axial magnetic flux is lower than in NS2016, $\Phi_{0}=6.3 \times 10^{18}$ Mx. More results about the numerical experiment will be provided in a follow-up paper (D. Nóbrega-Siverio et al. 2017, in preparation), where a deeper analysis of the theoretical aspects of the experiments is carried out.

3. Since we are interested in synthesizing Si IV for comparison purposes with the IRIS observations, spatial and spectral PSF (Gaussian) degradation of the theoretical data must be carried out to reduce the spectral profiles into the IRIS instrumental spatial and spectral resolution ( 0. " 33 and $26 \mathrm{m \AA}$ respectively). Finally, we convert the degraded line intensity from CGS units (erg $\mathrm{s}^{-1} \mathrm{~cm}^{-2} \mathrm{sr}^{-1}$ ) to IRIS photon count number $\left(\mathrm{DN} \mathrm{s}^{-1}\right)$ for direct comparisons using

$$
I_{I R I S}=I_{\mathrm{CGS}} \frac{A p w}{r^{2}} \frac{\lambda}{h c} \frac{1}{k},
$$

where $A=2.2 \mathrm{~cm}^{2}$ is the effective area for wavelengths between 1389 and $1407 \AA, p=0$." 167 is the spatial pixel size, $w=0$ !" 33 is the slit width, $r=3600 \cdot 180 / \pi$ is the conversion of arcsec to radians, $\lambda=1402.77 \AA$ is the wavelength of interest, $h$ is the Planck constant, $c$ is the speed of light, and $k=4$ is the number of photons per DN in the case of FUV spectra (see De Pontieu et al. 2014, for the instrumental specifications about IRIS).

\section{Observations: Results}

Along the observation, we are able to distinguish two main episodes of surge activity in the SST/CRISP maps with accompanying brightenings in the SJI 1400: one taking place between 08:14 and 08:39 UT and the other between 09:27 UT and 10:03 UT, which is the end of the coordinated observation. Both episodes seem to be related to the appearance of positive polarity patches within a pre-existing negative polarity region. In this paper, we focus on the second one since the IRIS spectral raster covers most of it in contrast to the first episode.

\subsection{Origin and Identification of the Surge and Burst}

As we said in the Introduction, surges are closely related to EFRs. For this reason, we looked for evidences of episodes of magnetic flux emergence and cancellation. Figure 2 illustrates the evolution of different integrated data within the solid 

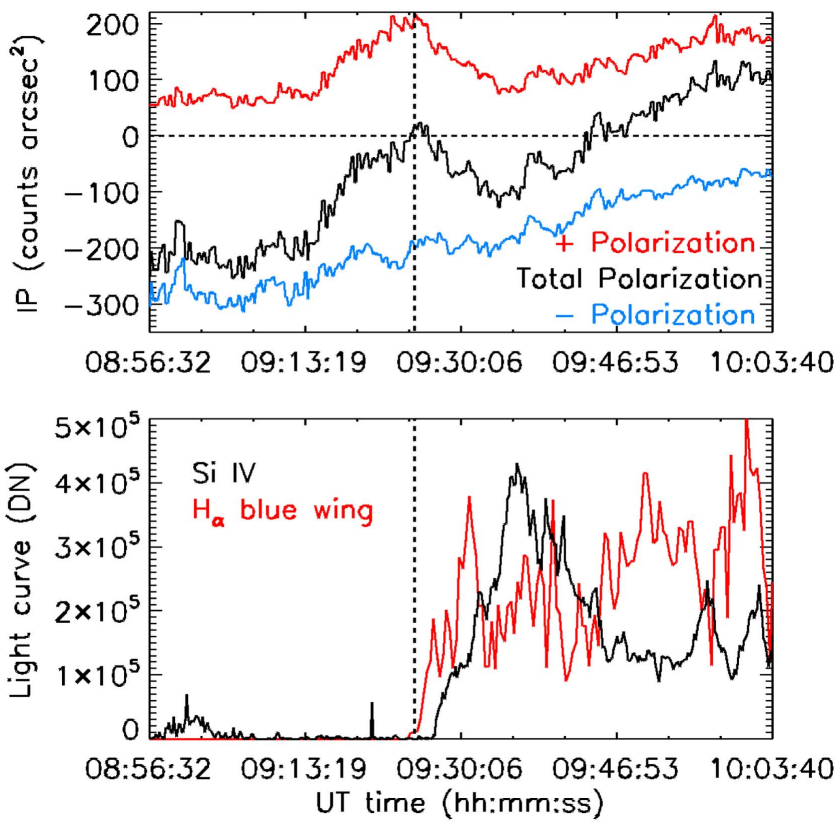

Figure 2. Curves obtained from the integration within the solid rectangle plotted in the three panels of Figure 1. Top: integrated polarizaton (IP), where the total polarization is shown in black, negative in blue, and positive polarization in red. The horizontal dashed line indicates the zero value for the polarization. Bottom: light curve of Si IV (black) and of the $\mathrm{H} \alpha$ blue wing (red). For both panels, the vertical dashed line marks the instant of the maximum polarization and the start of the brightenings.

rectangle of the three panels in Figure 1 (and the accompanying animation). The top panel of Figure 2 contains information about the magnetic flux; the black curve corresponds to the signed total flux and shows that, prior to the event, the region is dominated by the negative polarity. Around 09:13 UT, the positive polarity (red curve) increases up to four times its initial value, reaching the maximum value at 09:25 UT (vertical dashed line). This increase is due to positive polarity patches that appear within the rectangle in which we are integrating the flux. After the positive flux reaches its maximum, the total net flux decreases and, around 09:27 UT, first brightenings are observed in the SST H $\alpha$ images (see panel (C) of Figure 1) and also in $\mathrm{Ca}$ II. The brightness increase is also evident in the bottom panel of Figure 2, where we plot the light curve of the blue wing of $\mathrm{H} \alpha$ integrated in the solid rectangle of Figure 1. Cospatially with the SST brightenings and approximately 2 minutes later, brightenings are also visible in the IRIS SJI 1400: the Si IV burst, which is marked in panel (B) of Figure 1 by the R2 arrow, and whose corresponding integrated light curve is illustrated in the bottom panel of Figure 2 in black color. Simultaneously and around the same region where the burst originates, a surge is discernible as a dark and elongated structure consisting of small-scale threads, first in the blue wing and then in the red wing of $\mathrm{H} \alpha$ and $\mathrm{Ca}$ II (see $\mathrm{R} 1$ arrow in panel (C) of Figure 1). Those darkenings are visible in the images in wavelength positions corresponding to $\sim 20-60 \mathrm{~km} \mathrm{~s}^{-1}$. This surge has projected lengths ranging from a few megameters up to $8 \mathrm{Mm}$, while the projected width is around 1 or $2 \mathrm{Mm}$. After 25 minutes ( 09:52 UT), the activity of both the SiIV burst and the surge becomes weak; nonetheless, it is enhanced again almost at the end of the coordinated observation (10:03 UT). This may suggest a recurrent behavior similar to that observed for surges (Schmieder et al. 1995; Gaizauskas 1996; Jiang et al. 2007;
Uddin et al. 2012); and for IRIS bursts (Peter et al. 2014; Gupta \& Tripathi 2015).

\subsection{Spectral Properties of the Surge and Burst}

In this section, we analyze the surge and the accompanying burst, i.e., the structures labeled R1 and R2 in Figure 1. To that end, in panels (B) and (C) of Figure 1, we select an area (dashed rectangle) that coincides horizontally with the region covered by the IRIS raster, namely, -563 ". $2 \leqslant x \leqslant-558$ ". 0 and located vertically between 47 ! $^{\prime} 0 \leqslant y \leqslant 52$ ! 0 . Detailed maps of the intensity peak of Si IV $1402.77 \AA$ and $\mathrm{H} \alpha$ at $-46 \mathrm{~km} \mathrm{~s}^{-1}$ within that rectangle are shown as panels (A) and (B), respectively, in Figure 3. Furthermore, the figure contains spectral profiles for the various locations marked with symbols in panels (A) and (B): two for the surge (R1a and R1b, where we have averaged four spatial pixels in order to increase the signal-to-noise ratio), and three for the burst (R2a, R2b, and $\mathrm{R} 2 \mathrm{c})$. Those profiles are drawn as solid curves and correspond to the lines of Si IV $1402.77 \AA$ (left column) and $\mathrm{H} \alpha$ (right column). Furthermore, the left panels contain a Gaussian fit to the Si IV $1402.77 \AA$ profile (red dashed curve), and a reference average profile (black dashed-dotted line), which is calculated for the whole FOV and length of the observation.

On the other hand, and to extend the analysis, Figure 4 contains maps, also at $\sim 09: 39$ UT, of (A) the maximum intensity, $I_{\max }$, of the Si IV $1402.77 \AA$ profile; (B) the Doppler shift $v_{D} ;(\mathrm{C})$ the line width $\sigma$; and (D) the $\mathrm{R}$ (ed)-B(lue) asymmetry, which is the difference between the integral of the two wings of the profile in the same velocity ranges, in this case, between 50 and $80 \mathrm{~km} \mathrm{~s}^{-1}$ (see De Pontieu et al. 2009; Martínez-Sykora et al. 2011; Tian et al. 2011, for further details about the R-B asymmetry calculation). The variables $v_{D}, \sigma$, and $\mathrm{R}-\mathrm{B}$ are obtained from the Gaussian fit centered at the spectral position of the peak of the profile. The lower four panels contain the same variables but after being binned twice in each direction, thus getting a better signal-to-noise ratio and facilitating the identification of the surge in Si IV. An animation of this figure is available online.

\subsubsection{R1: the Surge}

The region selected for Figure 3 contains about one-third of the surge (R1), which is identifiable as a dark structure in the $\mathrm{H} \alpha$ image between $y=47^{\prime \prime}$ and $y=50^{\prime \prime}$. The $\mathrm{H} \alpha$ profiles R1a,b underneath show a dark blue wing with respect to the reference profile. Those two profiles correspond to two different locations of the surge: the brightest point of Si IV $1402.77 \AA$ within the surge (blue diamond, hereafter R1a), and a fixed position around $x=-562$ ". 5 and $y=48$.' 5 (blue cross, $\mathrm{R} 1 \mathrm{~b})$. In both locations, we can identify significant emission in Si IV that, as far as we know, has not been reported before in the literature. The main properties of this region concerning Si IV are as follows.

1. The emission in Si IV is sporadic and we see it mostly when the surge appears in the blue wing of $\mathrm{H} \alpha$, in particular, between 09:34 UT and 09:41 UT (see the animation of Figure 3).

2. The SiIV intensity within the surge is weak in comparison to the burst, which could be the reason why it has not been reported before. This is evident in panels (A) and (E) of Figure 4 if we compare the values within the red contour (the burst) with the ones inside the blue 

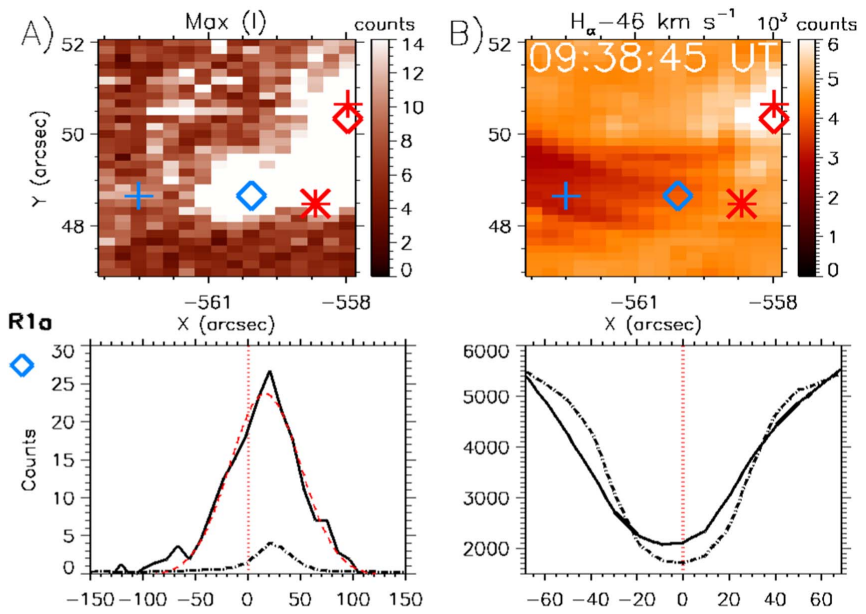

R1b
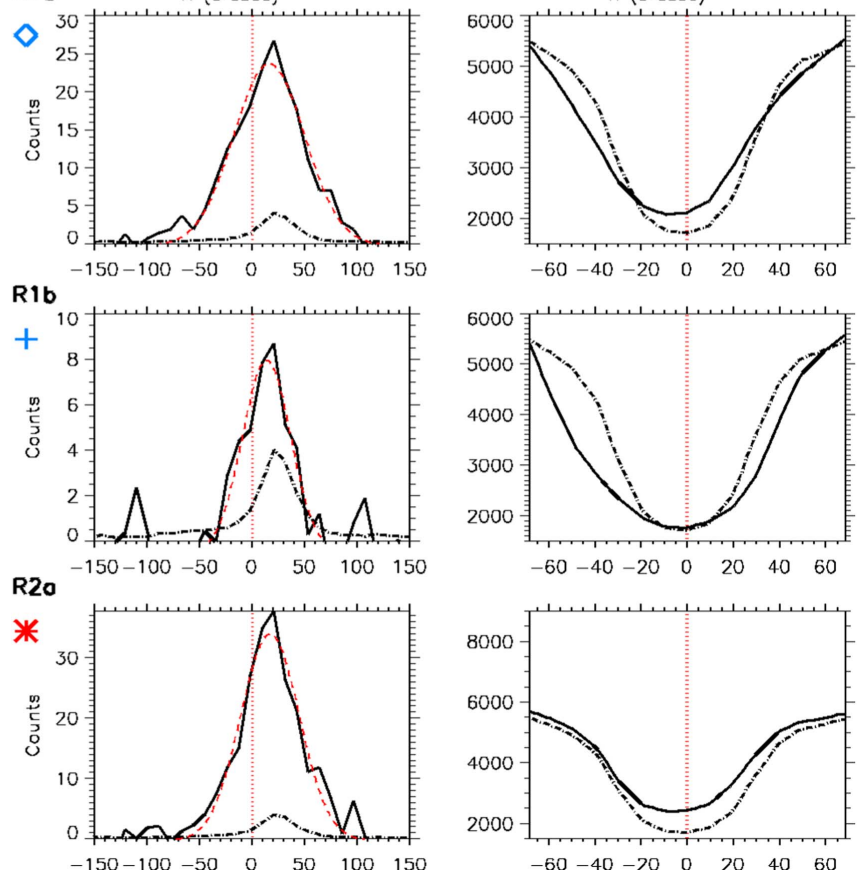

R2b

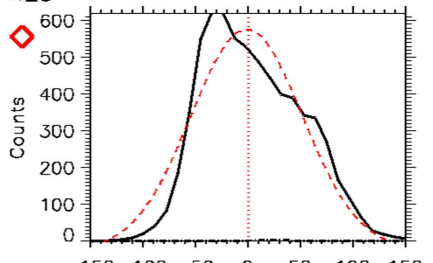

$-150-100-50 \quad 0 \quad 50 \quad 100 \quad 150$

R2c
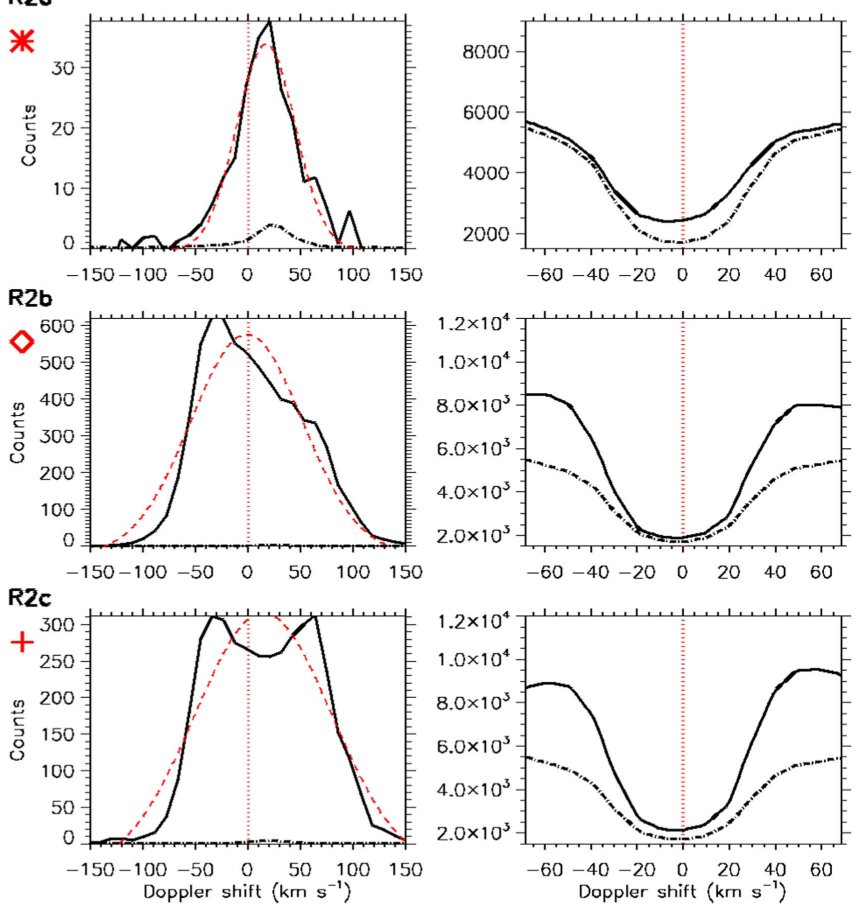

Figure 3. (A) Raster intensity peak map of the Si IV $1402.77 \AA$ profile. (B) Image at the blue wing of $\mathrm{H} \alpha\left(-46 \mathrm{~km} \mathrm{~s}^{-1}\right)$. Various locations of regions R1 and R2 are indicated by blue and red symbols, respectively, and their corresponding profiles are shown below panels (A) and (B). The left column illustrates Si IV $1402.77 \AA$ profiles (black solid line), the Gaussian fit to them (red dashed curve), and the reference average profile (black dashed-dotted line). The right column illustrates the $\mathrm{H} \alpha$ profiles in the various regions (solid line) and the reference average profile (dashed-dotted line). For R1 panels, we have averaged four pixels in order to increase the signal-to-noise ratio of this region. The animation of this figure shows the time evolution of the profiles of each region from $\sim 09: 30$ UT until $\sim 09: 51$ UT.

(An animation of this figure is available.)

contour (the surge). During the event, the ratio between the peak of the Si IV profile and the one of the reference profile (black dashed-dotted line in Figure 3) is on
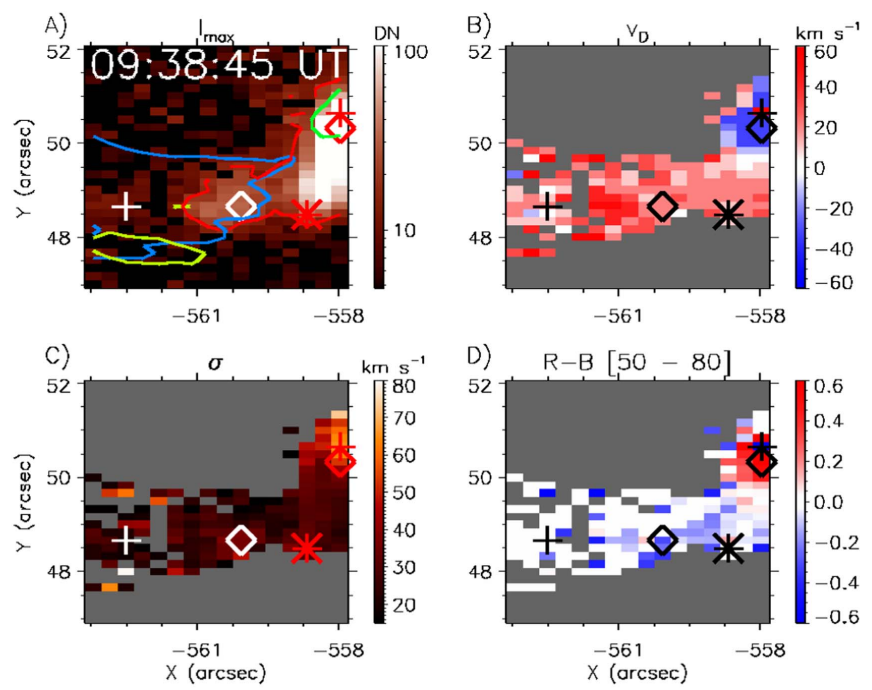

Binned version
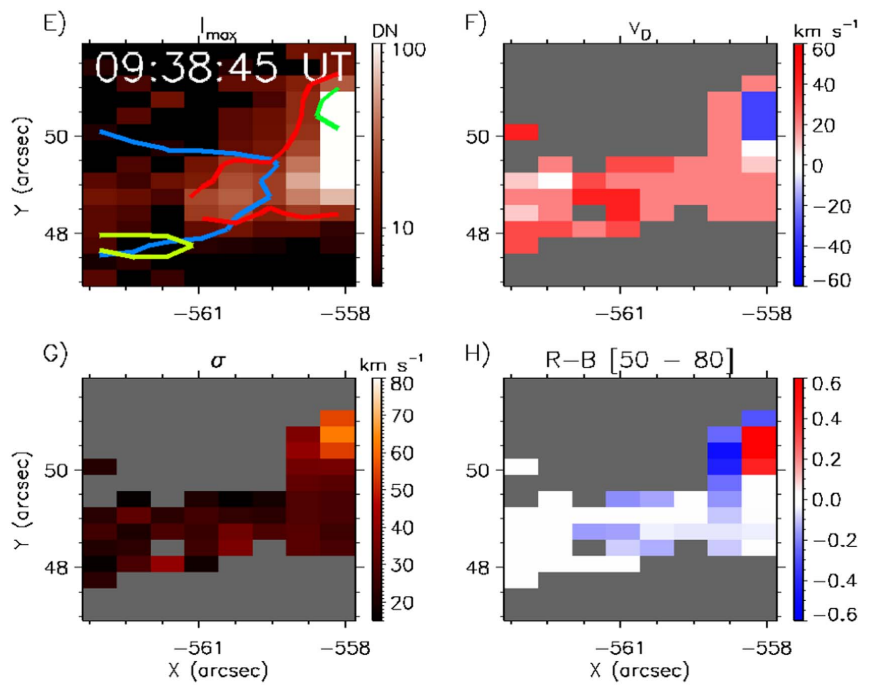

Figure 4. Four upper panels are maps of various features of the Si IV spectral profiles. (A) Raster intensity peak of the Si IV 1402.77 $\AA$ profile at $\sim 09: 39$ UT with overlying contours indicating the burst (red contour, where $I_{\max }>16 \mathrm{DN}$ ); the EB (green); the surge in the $\mathrm{H} \alpha$ blue wing (blue); and in the red wing (yellow). (B) Doppler shift $v_{D}$ in $\mathrm{km} \mathrm{s}^{-1}$, where the spectral position corresponding to the maximum intensity is used as the line centroid; (C) line width $\sigma$ in $\mathrm{km} \mathrm{s}^{-1}$; and (D) R-B asymmetry between 50 and $80 \mathrm{~km} \mathrm{~s}^{-1}$ normalized to the peak intensity. A gray color mask is overplotted in panels (B)-(D) in places outside the burst and surge, and where the intensity is below 5 counts and $\sigma$ is below $16 \mathrm{~km} \mathrm{~s}^{-1}$. The four lower panels show the same quantities after a spatial binning by a factor of two in each direction. The animation of this figure contains the time evolution of the panels from $\sim 09: 30$ UT until $\sim 09: 51$ UT.

(An animation of this figure is available.)

Table 1

Temporal Averages of Si IV $1402.77 \AA$ for (1) Peak Intensity $I_{\text {peak }}$ with Respect to That of the Reference Profile $I_{\text {peak, } 0}$, Doppler Shift $v_{D}$, and Line Width $\sigma$

\begin{tabular}{lccc}
\hline \hline Region & $\left\langle I_{\text {peak }} / I_{\text {peak }, 0}\right\rangle$ & $\left\langle v_{D}\right\rangle\left(\mathrm{km} \mathrm{s}^{-1}\right)$ & $\langle\sigma\rangle\left(\mathrm{km} \mathrm{s}^{-1}\right)$ \\
\hline R1a & $5.2 \pm 1.4$ & $20.2 \pm 10.4$ & $22.9 \pm 5.3$ \\
R1b & $1.9 \pm 0.5$ & $22.7 \pm 20.5$ & $29.6 \pm 9.4$ \\
\hline
\end{tabular}

average around 5.2 in R1a and 1.9 in R1b (see Table 1), with maximum values of 8.9 and 2.7 respectively.

3. Given its definition, R1a, the brightest Si IV location within the surge, moves with time, but we have found 
that it is mostly located near the region that we have called the footpoint of the surge in Section 2.1 (see the animation of Figure 3). It can also be seen that R1a is a small-scale brightening of a few pixels at most. This suggests that IRIS does not completely resolve the spatial structure of R1a. The location of the brightest Si IV emission that is near the footpoints is reproduced in the numerical experiments and could be a characteristic feature of the surges. In fact, in the experiments we also provide a possible reason why $\mathrm{R} 1 \mathrm{a}$ is sometimes not so close to the footpoints (Section 4.2.1).

4. In the observed surge, Si IV shows mainly redshifted profiles (see panels R1a and R1b in Figure 3 and panels (B) and (F) of Figure 4). In order to illustrate this quantitatively, we calculate average values of $v_{D}$ for the two regions $\mathrm{R} 1 \mathrm{a}$ and $\mathrm{R} 1 \mathrm{~b}$ when the Si IV intensity is larger than 5 counts and $\sigma$ is at least $16 \mathrm{~km} \mathrm{~s}^{-1}$, i.e., when the Si IV signal is greater than the reference average profile. The results are shown in Table 1. We see that the Si IV emission within the surge shows redshifts with an average value around $20-23 \mathrm{~km} \mathrm{~s}^{-1}$. (Note that the relative Doppler velocity is blueshifted around $2 \mathrm{~km} \mathrm{~s}^{-1}$ only with respect to the reference profile.)

5. Within the surge, the line width $\sigma$ is around $23-29 \mathrm{~km} \mathrm{~s}^{-1}$ (see panel (C) in Figure 4 and the corresponding average in Table 1). Those values are approximately a factor of 4 greater than the corresponding value of the width just for thermal broadening $\left(\sigma_{\mathrm{th}}=6.86 \mathrm{~km} \mathrm{~s}^{-1}\right.$ taking the temperature formation peak at $\log (T)=4.90$ in statistical equilibrium). We calculate the nonthermal line broadening $\sigma_{n t}$ following De Pontieu et al. (2015) with

$$
\sigma_{n t}=\sqrt{\sigma^{2}-\sigma_{\mathrm{th}}^{2}-\sigma_{\mathrm{inst}}^{2}},
$$

where $\sigma_{\text {inst }}=3.9 \mathrm{~km} \mathrm{~s}^{-1}$. The obtained nonthermal widths, 21.45 and $28.52 \mathrm{~km} \mathrm{~s}^{-1}$ for R1a and R1b, respectively, are on the upper range of the typical values for an active region (see De Pontieu et al. 2015); nevertheless, we would need more Si IV observations to carry out a statistical analysis in order to know whether this is an intrinsic characteristic of the surges or not.

6. Concerning panel (D) of Figure 4, we find some R-B asymmetry mainly to the blue side in the surge footpoints. This is more evident in panel $(\mathrm{H})$ of the figure, which contains a version of panel (D) spatially binned by a factor two in both directions. The found R-B asymmetry implies that a small fraction of the surge plasma is moving with velocities greater than $50 \mathrm{~km} \mathrm{~s}^{-1}$ relative to the profile peak.

\subsubsection{R2: the Burst}

The other region we have analyzed is the part of the burst (R2) for which we have IRIS spectral profiles. The three lower rows of Figure 3 show the profiles in three different locations: the brightest $\mathrm{Si}$ IV point between $x=-558$.' 67 and $x=$ -557 !' 97 , which corresponds to locations within the burst close to the surge footpoints (red asterisk, hereafter R2a); at the location within the burst that has the maximum Si IV emission (red diamond, $\mathrm{R} 2 \mathrm{~b}$ ), and at the brightest point in the $\mathrm{H} \alpha$ wings at $46 \mathrm{~km} \mathrm{~s}^{-1}$ within the area of the Si IV burst (red cross, R2c). In the following, we describe the main properties of this region.

1. R2a can be considered the transition between the surge and the burst. In this region, the Si IV profiles repeatedly change in time between being blueshifted and redshifted, with small Doppler velocities and line widths of $\pm 30 \mathrm{~km} \mathrm{~s}^{-1}$ (see the accompanying movies of Figures 3 and 4). In Si IV, this region is slightly brighter than R1, but weaker than the following ones. The latter can be said also for $\mathrm{H} \alpha$, which is characterized by showing some enhancement, both in the wings and core, but not at the same level as R2b and R2c.

2. Regarding R2b and R2c, the Si IV spectra are characterized by multicomponent profiles, see the corresponding panels in Figure 3; the blue component of the profiles is usually the brightest one, as shown in panel (B) of Figure 4, where the peak of the profile is blueshifted; the profiles are broad, see panel (C), where $\sigma>60 \mathrm{~km} \mathrm{~s}^{-1}$, and they show strong asymmetry to the red, see panel (D). The polarization map in Figure 1 reveals that R2c mostly coincides with the location where the two underlying opposite polarities collide. This situation is often interpreted in the observational literature by assuming that the two components of the Si IV profiles correspond to bidirectional flows due to magnetic reconnection. Further evidence about magnetic reconnection and ejection of plasmoids is provided by Rouppe van der Voort et al. (2017) who analyze the same IRIS and SST observations but include spectrally resolved Ca II $\mathrm{K}$ imaging from the new CHROMIS Fabry-Pérot instrument at the SST.

During the event, the brightest Si IV (R2b) is usually located close to the brightest $\mathrm{H} \alpha$ wing (R2c) in the upper part (solar $Y$-axis) of the burst in Si IV, and they can even overlap on a few occasions. Interestingly, between 09:45:30 and 09:46:10, when the surge is visible in the red wing of $\mathrm{H} \alpha, \mathrm{R} 2 \mathrm{~b}$ moves down in the $Y$-axis approximately $2^{\prime \prime}$, while R2c holds in the same position. This may indicate motion of plasma emitting strongly in Si IV. The two-component profiles described above and this motion of the brightest point in Si IV within the burst are addressed in Section 4.2.2.

We have also carried out a brief study of the other spectral lines observed by IRIS and found that R2b and R2c show absorption features superimposed on the Si IV $1393.76 \AA$ line that correspond to Ni II $1393.33 \AA$ redshifted around $13 \mathrm{~km} \mathrm{~s}^{-1}$. We looked for further evidence in the IRIS spectra and also detected absorption of Ni II $1335.20 \AA$ in the C II profile. Similar absorption features have been reported in IRIS lines by other authors like Schmit et al. (2014), Peter et al. (2014), Kim et al. (2015), Vissers et al. (2015), and Tian et al. (2016), which indicate that there is overlying dense and cold plasma on top of the Si IV source. Concerning the other IRIS lines, there are brightenings in the wings of $\mathrm{Mg} I \mathrm{~h} \mathrm{~h}$ and $\mathrm{k}$ lines with small enhancement in the cores, which is akin to what was described by Tian et al. (2016) about IRIS bursts and point to heating of chromospheric plasma. There is also evidence of emission in the triplet of subordinate lines of Mg II at 2798.75 and $2798.82 \AA$, indicating an abrupt temperature increase in the lower chromosphere (see Pereira et al. 2015). The IRIS profiles do not show 

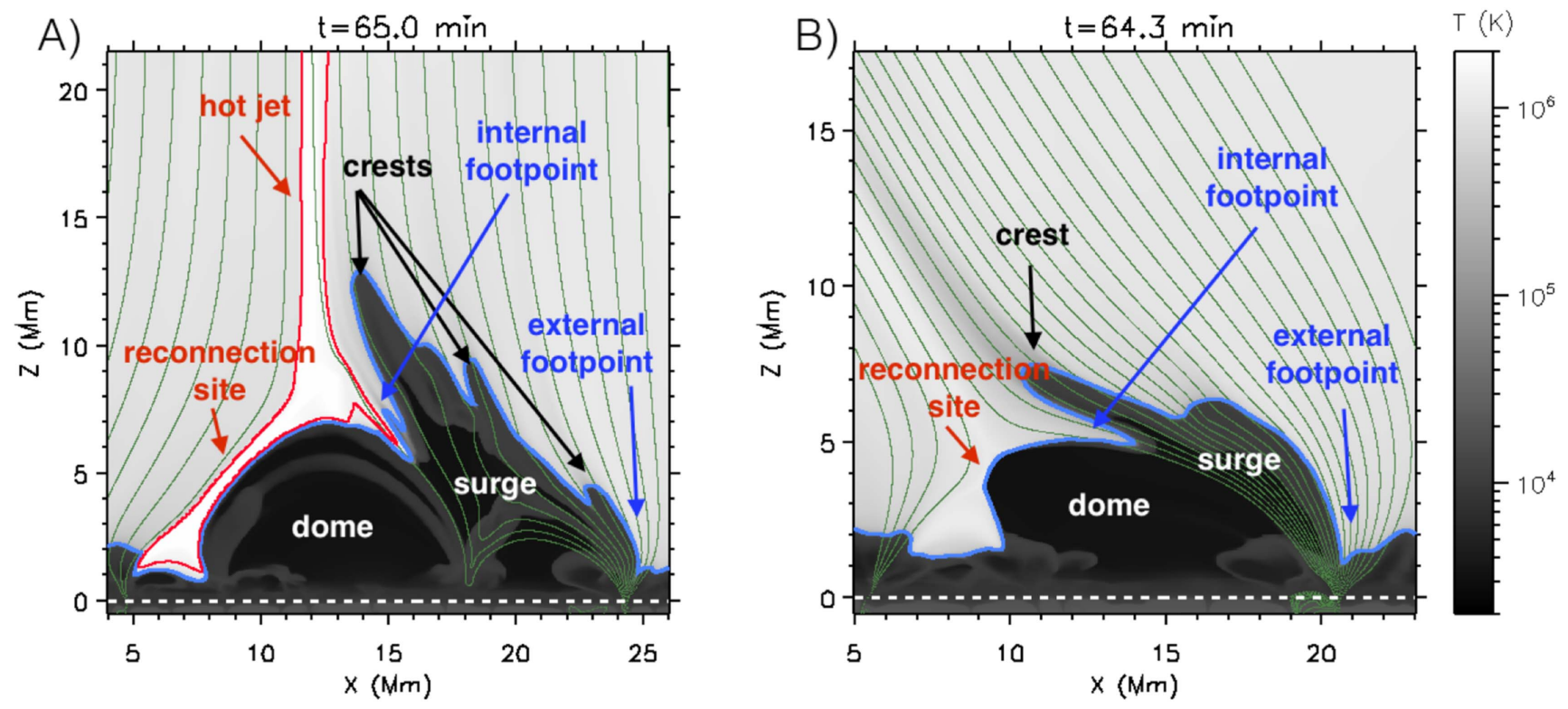

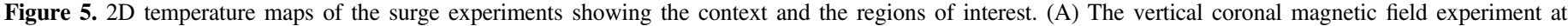

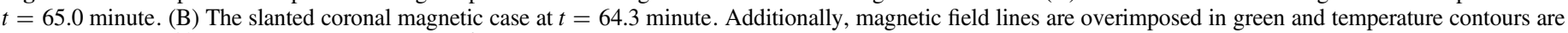
added for $T=10^{4.9} \mathrm{~K}$ (blue) and $T=1.2 \times 10^{6} \mathrm{~K}$ (red).

the forbidden O IV 1401.2 and $1399.8 \AA$ lines, so we cannot use them for density diagnostics as carried out by Olluri et al. (2013a), Polito et al. (2016), or Martínez-Sykora et al. (2016). There is no evidence, either, of Fe XII at $1349 \AA$, which forms around 1.5 MK. The lack of O IV and Fe XII is likely due to the very short exposure time of this observation $(0.5 \mathrm{~s})$ since those lines are fainter and require longer exposure times (De Pontieu et al. 2014).

With respect to the spectra obtained by SST, in Figure 3, we see enhanced wings in the $\mathrm{H} \alpha$ profiles both in $\mathrm{R} 2 \mathrm{~b}$ and in $\mathrm{R} 2 \mathrm{c}$, which resemble the profiles found by Kim et al. (2015) in IRIS bursts. In the associated movie, we can see that the $\mathrm{H} \alpha$ profiles can show extremely enhanced wings, while the core is mostly keept unperturbed, as in EB profiles.

\section{Numerical Experiment: Results}

In this section, we analyze synthetic profiles from the numerical experiments introduced in Section 2.2 in order to compare them with the previously described observations, thus attempting to provide a theoretical understanding of the phenomena studied. The layout of this section is as follows: (1) a general description of the time evolution of the experiments, and (2) the synthesis and comparison with the IRIS observations.

\subsection{A Brief Description of the Time Evolution}

The general features of the time evolution of these experiments are qualitatively similar to those described by NS2016. In the present paper, we focus on the advanced phases of the evolution, when the magnetized emerged plasma that has expanded into the solar atmosphere collides with the pre-existing coronal field, and a surge is obtained as an indirect consequence of magnetic reconnection processes.

Figure 5 shows the context of the numerical simulations through a temperature map for each experiment: panel (A), the vertical experiment at $t=65.0$ minute, and panel (B), the slanted one at $t=64.3$ minute. In panel (A), we can see the emerged plasma with a dome shape between $8 \leqslant x \leqslant 18 \mathrm{Mm}$; a coronal hot jet, with a classical Eiffel-tower shape (see the red contour) and maximum temperatures of $T \sim 2-3 \mathrm{MK}$; and the surge, which is the cool and elongated structure, as in NS2016, located on the right side of the dome. The surge has been produced by a peeling-like mechanism during the nonstationary magnetic reconnection process. This process drags away and ejects plasma from the dome, sometimes as plasmoids, giving rise to structures that resemble crests. The inner part of the surge is composed of elongated thread-like structures of different density that are a consequence of the collision and subsequent merging of plasmoids with the surge. Besides that, there is also a detachment mechanism that splits and separates the surge from the emerged dome (see the details about this process in NS2016). This occurs at a height of around 5 or $6 \mathrm{Mm}$ on the side of the surge closest to the reconnection site, referred to in the following as the internal side of the surge. Consequently, we call that region the internal footpoint of the surge (as marked by an arrow in the figure). Another interesting region for the comparison with the observations is the base of the external region of the surge: we call this the external footpoint of the numerical surge, and it is marked by another arrow in the figure. In panel (B), the slanted experiment, the emerged dome is located at $10 \leqslant x \leqslant 20 \mathrm{Mm}$, and the coronal jet has lower temperatures $(T<1.2 \mathrm{MK})$. In this case, the surge does not end up totally detaching from the dome, which could be a consequence of the magnetic field configuration in the corona. In the follow-up paper (D. Nóbrega-Siverio et al. 2017, in preparation), an in-depth study of the physics taking place in this phenomenon is carried out, including the role of the entropy sources and the consequences of the NEQ ionization of silicon and oxygen for the emissivity.

\subsection{Analysis of the Synthetic Spectra}

With a view to identifying the different observational features explained in the paper (Section 3.2) with structures and phenomena in the experiments, in the following, we 

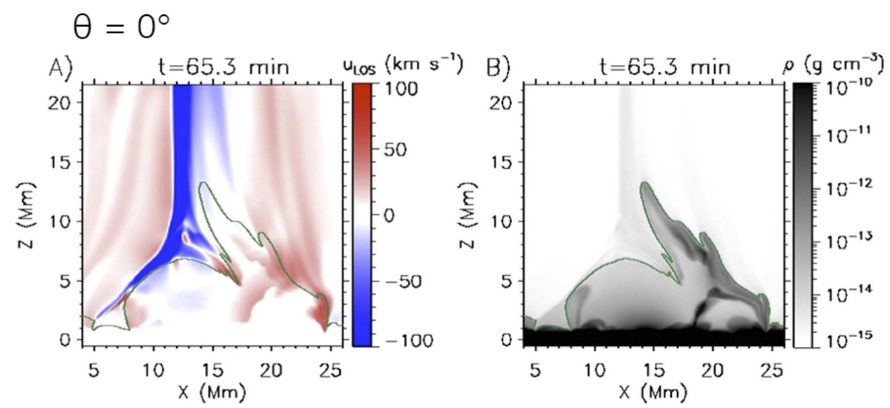

$$
\theta=-15^{\circ}
$$
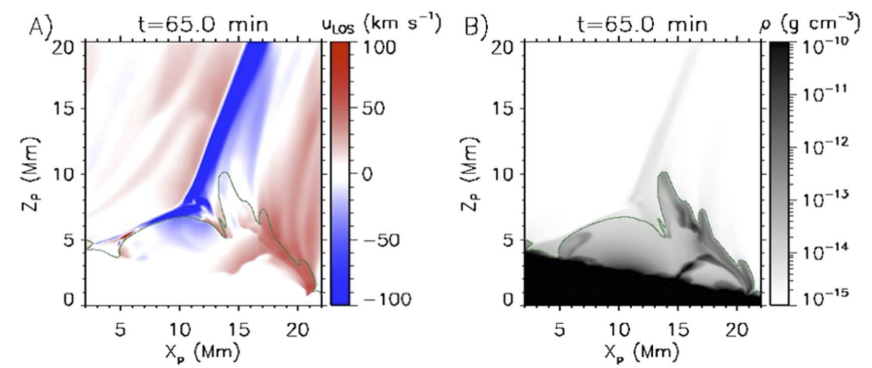

$\theta=15^{\circ}$
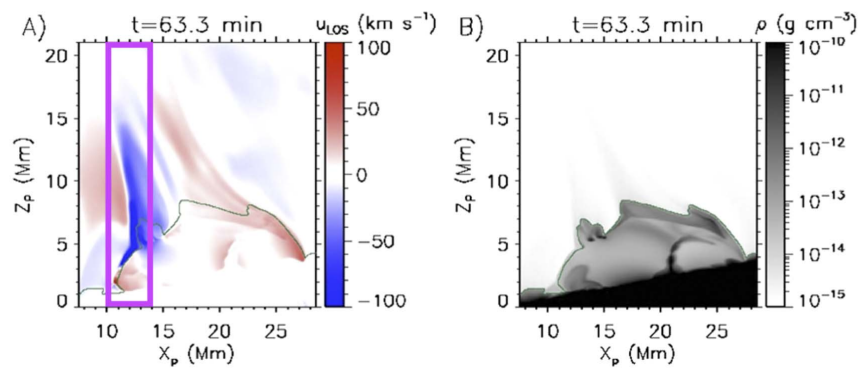
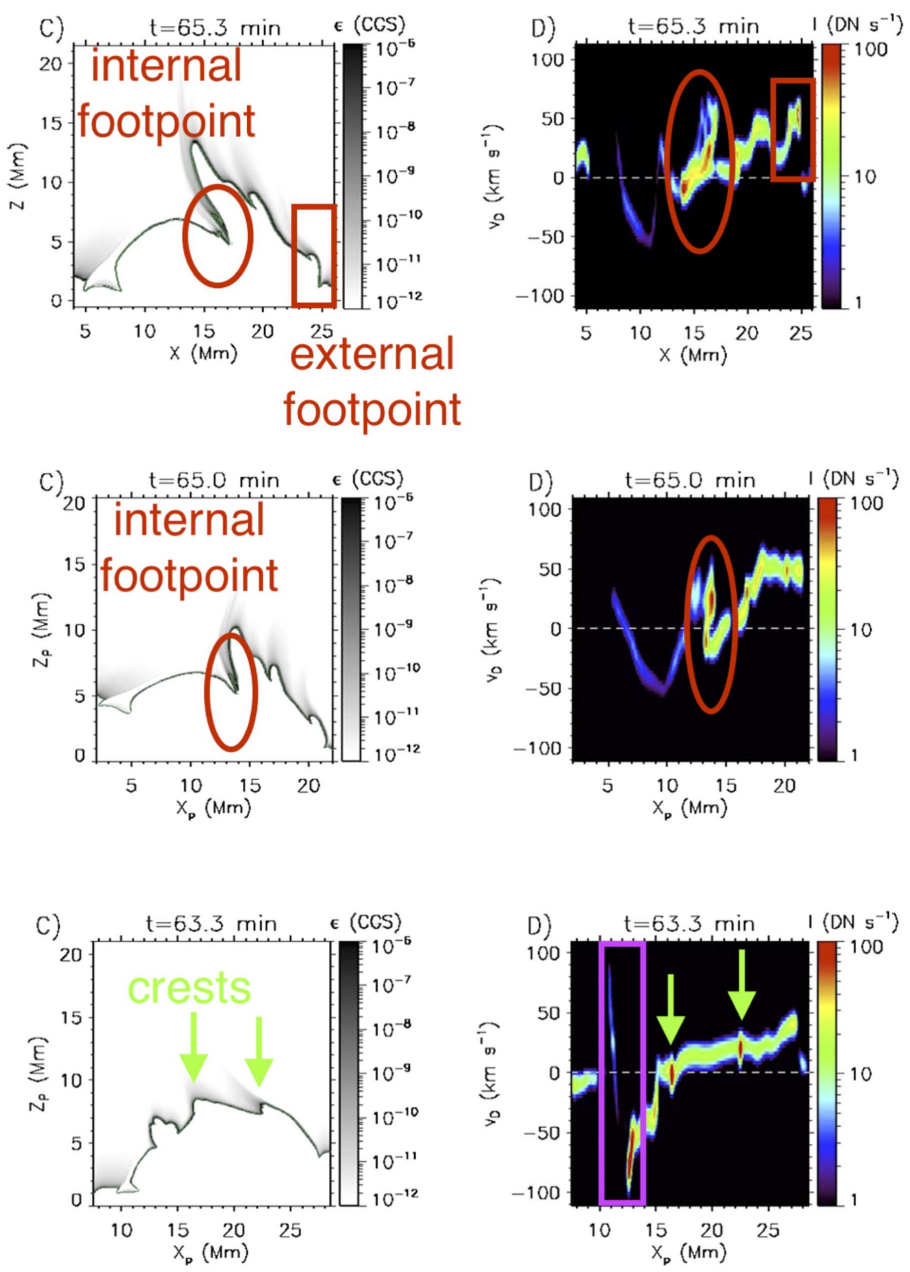

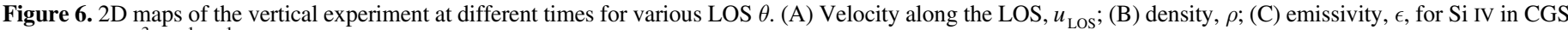

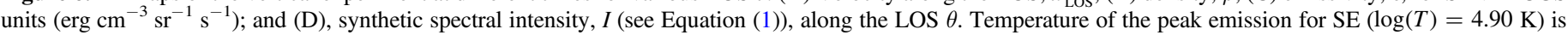

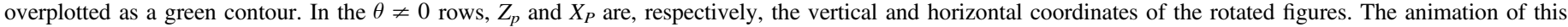

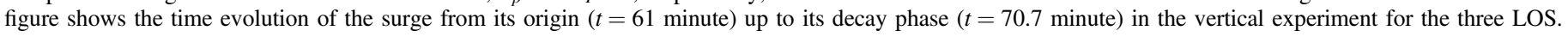

(An animation of this figure is available.)

analyze the synthetic Si IV $1402.77 \AA$ spectra obtained from the numerical data. The surge in the experiments produces spectral features that resemble those from Region R1 in the IRIS Si IV observations (Section 3.2.1). In turn, the region near the magnetic reconnection site, whose location is marked in Figure 5 for both experiments, produces spectral features that resemble the burst (R2) described in Section 3.2.2. In order to establish the relation between the numerical and observational features for the surge and burst, we use Figures 6 and 7, which correspond to the vertical and slanted magnetic field experiments respectively. The different rows correspond to different inclination angles for the LOS. Exploring different inclinations is important since they impact the obtained intensities as seen in the following. The panels in each row contain (A) the velocity along the LOS, $u_{\mathrm{Los}}$; (B) the density, $\rho$; (C) the Si IV emissivity, $\epsilon$; and (D) the synthetic spectral intensity, $I$, derived from Equation (1). In the $\theta \neq 0$ panels, $x_{P}$ and $z_{P}$ are, respectively, the horizontal and vertical coordinates of the rotated figures. Note that, to facilitate the explanation, we are choosing somewhat different times for each row. Animations of the Figures 6 and 7 are provided online.

\subsubsection{The Surge}

In the following, we separately analyze various characteristic regions of the surge.

(a) The internal footpoint. For the vertical field experiment (Figure $6, \theta=0^{\circ}$ and $\theta=-15^{\circ}$ ), in panel (D) at $x \sim 16.5 \mathrm{Mm}$ and $x_{P} \sim 13.5 \mathrm{Mm}$, respectively, there is a typical spectrum of the internal footpoint obtained through integration along an LOS near and parallel to the transition region at the internal boundaries of the surge. Regarding the slanted experiment (Figure 7, $\theta=0^{\circ}$ row), in panel (D), approximately between $11 \leqslant x \leqslant 14 \mathrm{Mm}$, we can see also a clear example of the spectrum emanating from the internal footpoint of the surge. This region is composed of plasma strongly emitting in Si IV, (see panels C) that during the event moves from the reconnection site to the internal footpoint. The plasma here is mostly descending due to the enhanced pressure gradient that pushes it down when going through the post-shock region (see NS2016); as a consequence, the spectra show mainly redshifted profiles. We note that the internal footpoint is usually brighter than the rest 
$\theta=0^{\circ}$
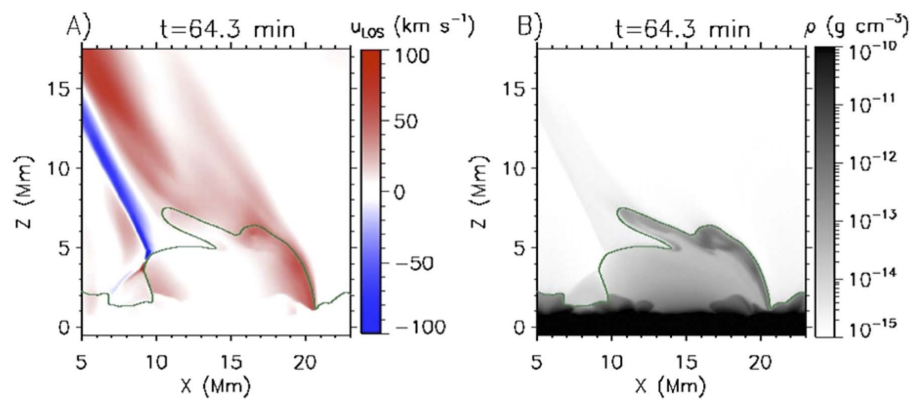

$\theta=-15^{\circ}$
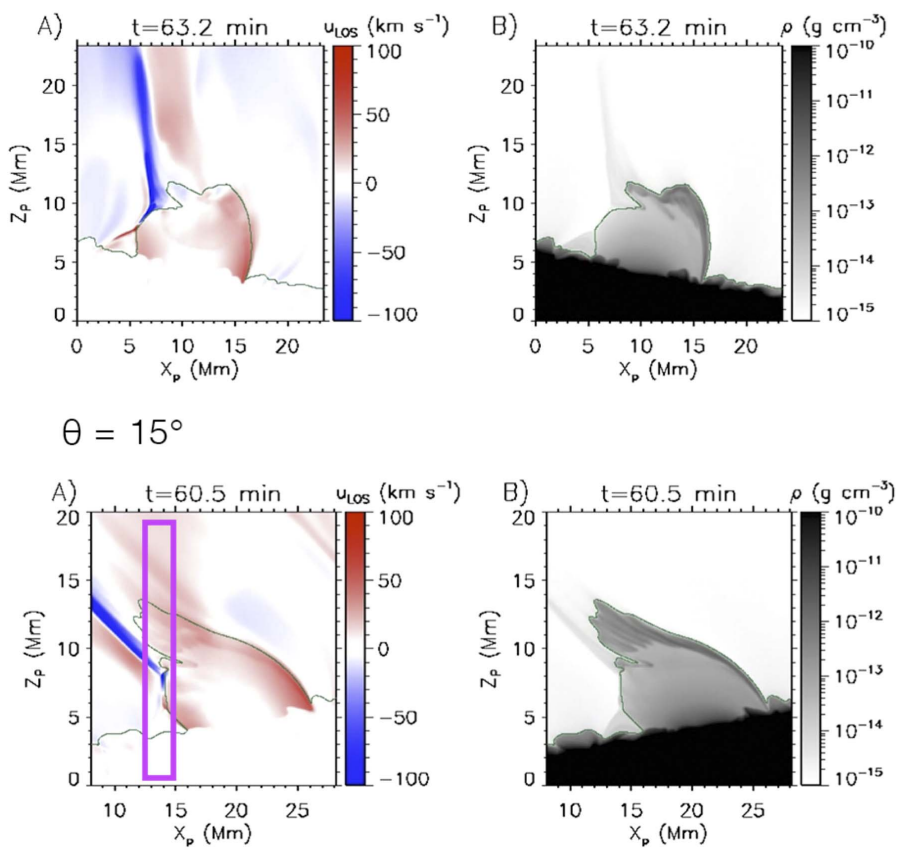
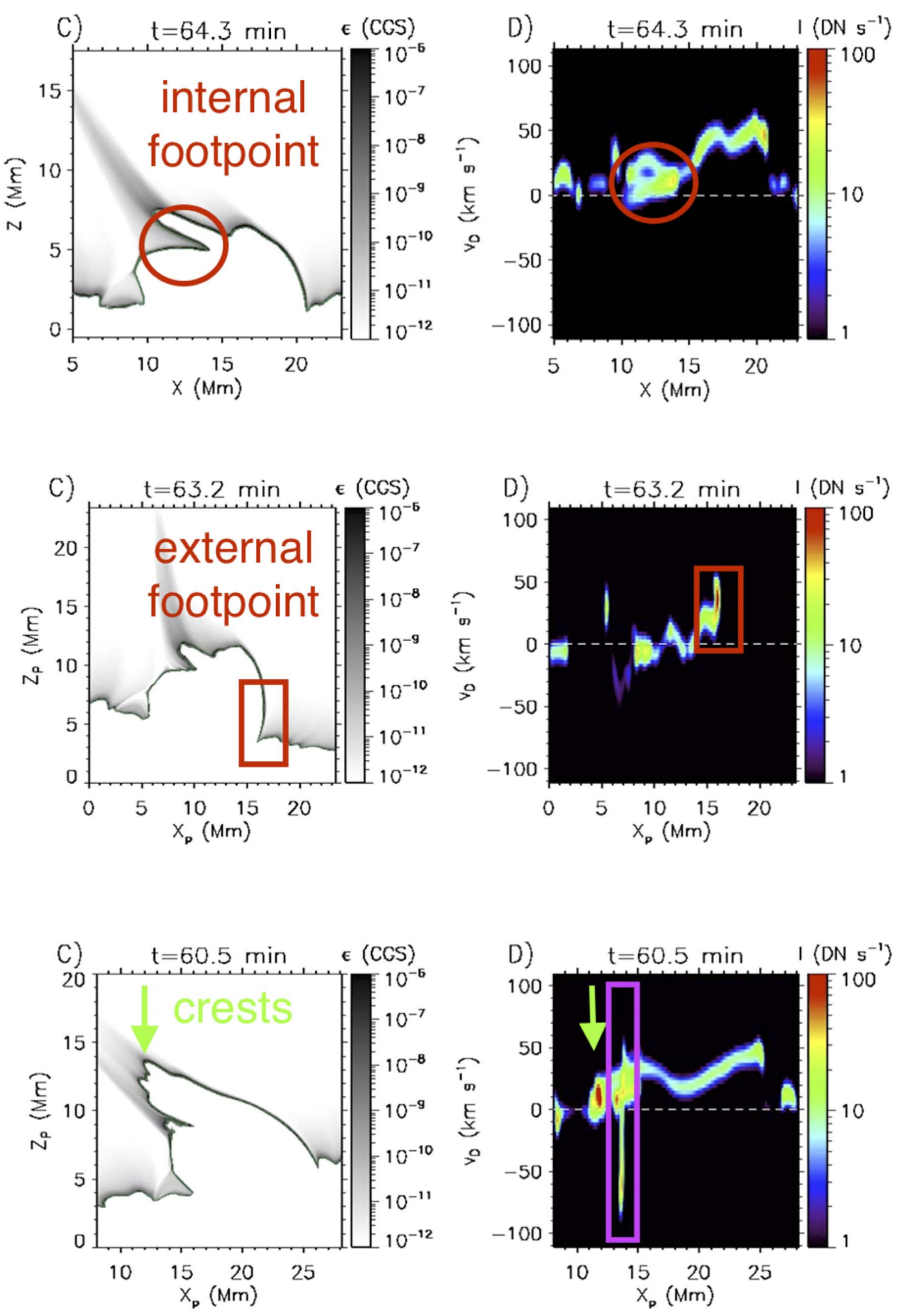

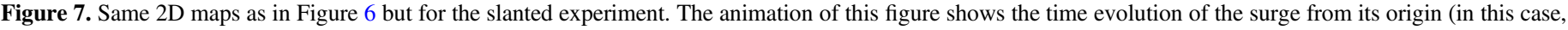
$t=58$ minute) up to its decay phase ( $t=68$ minute) for the three LOS.

(An animation of this figure is available.)

of the surge. In the animations of the vertical experiment, we can see that the intensity of this region varies depending on the LOS and can be greatly enhanced when integrating parallel to the transition region of the internal footpoint.

The features mentioned above fit with the results of the IRIS observation (Section 3.2.1) for region R1a (the brightest region within the surge), which is mostly located around the observational footpoints. The deviations from this behavior are explained in the subsequent items.

(b) The external footpoint. Figure 6 shows that in that region (marked in the figure by a red rectangle) we can also find brightenings with intensities between 30 and $112 \mathrm{DN} \mathrm{s}^{-1}$. The intensity depends again on the geometry, i.e., it is enhanced when the parallel direction of the transition region of the surge coincides with the LOS. This dependence can be checked by looking at the three different $\operatorname{LOS} \theta$ of the same figure. In this region, the Doppler shift $v_{D}$ has values around $20-60 \mathrm{~km} \mathrm{~s}^{-1}$ to the red. A similar result is found for the external footpoint of the slanted experiment (see, e.g., the region marked with the circle in the row $\theta=-15^{\circ}$ of Figure 7).
Concerning the observations, the above result strengthens the conclusion of Section 3.2.1 that bright Si IV points within the surge are mostly located at its footpoints. Note that in the current observations we cannot distinguish whether the brightenings of R1a come from the internal or external footpoints.

(c) The crests. Representative cases for the spectra of the surge crests can be found in the $\theta=15^{\circ}$ row of the vertical experiment (marked with green arrows in Figure 6), which shows: (a) a crest in its ascending phase approximately at $x_{P}=15 \mathrm{Mm}$, with intensities around $40 \mathrm{DN} \mathrm{s}^{-1}$ and blueshifted; (b) a much brighter one with blue and redshifted components although with small velocities $\left(x_{P}=17 \mathrm{Mm}\right)$; and (c) the last one, which is also bright but only shows redshift $\left(x_{P}=22.5 \mathrm{Mm}\right)$. At $x_{P}=13.0 \mathrm{Mm}$, the magnetic reconnection process is leading to another crest as a result of the ejection of plasma upwards with large velocities (see panel (A)). Regarding the slanted experiment (Figure 7), the $\theta=15^{\circ}$ row shows a crest example between $11 \leqslant x_{P} \leqslant 13 \mathrm{Mm}$. In this case, we find that before $t=59.0$ minute, the crest is a bright feature on the blue side of the spectrum with 
Vertical Experiment Slanted Experiment

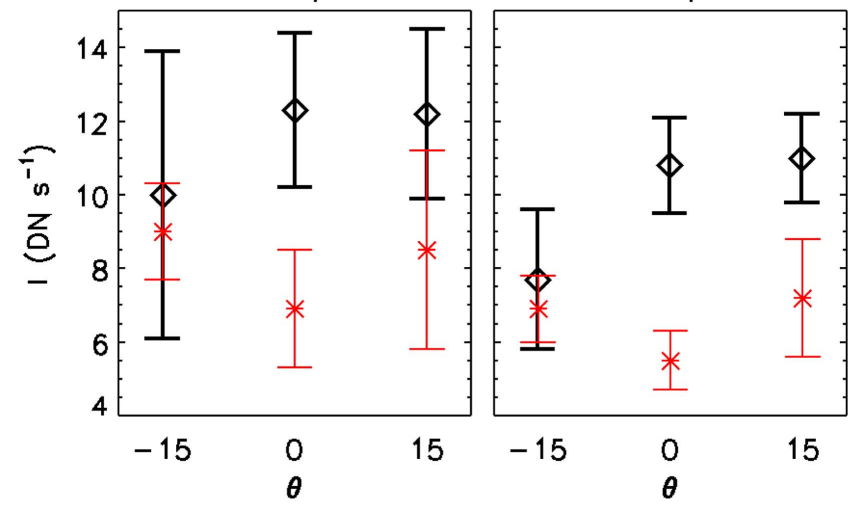

Figure 8. Average intensities for the three different LOSs of the less bright regions of the surge (black diamonds) and of the quiet transition region (red asterisks) for both experiments. The error bars are obtained from the standard deviation.

absolute $v_{D}$ of $<30 \mathrm{~km} \mathrm{~s}^{-1}$; nonetheless, as time advances on (e.g., $t=60.5$ minute), the whole surge falls down and the crest then shows redshifted profiles with velocities on the same order. At later stages of the evolution, the crest moves to the right side of the dome and it is not easy to isolate it to integrate the emissivity without going through other interesting sites like the magnetic reconnection region.

With respect to the comparison with the observations, in Section 3.2.1, we argued that R1a, which was defined as the pixel with the brightest Si IV emission within the surge, although mostly located near the footpoints, can sometimes be found further away. Now we realize that those excursions from the observational footpoint region can correspond to instants in which the brightest $\mathrm{Si}$ IV emission is produced in the crests of the surge instead of in the footpoints themselves.

(d) The rest of the surge. Let us focus now on the less bright regions of the surge. In Figures 6 and 7, we see that the lowest intensities within the surge are $\sim 10 \mathrm{DN} \mathrm{s}^{-1}$. We would like to explore whether even those regions are intrinsically brighter or not than an average transition region. In order to show this quantitatively, we have carried out a statistical analysis in quiet transition regions, i.e., places far away from the locations affected by the magnetic reconnection and the subsequent surge. In those regions, we calculate the average peak intensity during 10 minutes; in the less bright regions of the surge, we average the minimum of the peak intensities within the numerical surge, also during 10 minutes. The resulting intensities and their standard deviations are shown in Figure 8 for the three LOSs used in the experiments. The diamonds correspond to the less bright regions of the surge; the asterisks correspond to the quiet transition region. Comparing those values, one could tentatively conclude that even the less bright regions of the surge seem to be slightly brighter than an average transition region; nonetheless, that statement is not fully conclusive given the large size of the standard deviation in some of the cases.

Concerning the IRIS observations, the fixed position R1b (Section 3.2.1) showed that in Si IV the surge was brighter than the average by a factor of 1.9 (see Table 1). In fact, in Figure 4 and the associated movie, the whole surge is above the peak intensity of the average profile. In the numerical experiments, the comparison between intensities from a quiet transition region and the less bright regions in the surge tentatively appears to be in accordance with the observations, although more statistics from other experiments would be needed for a stronger conclusion.

\subsubsection{The Burst}

After analyzing the spectra of the surge, in this section, we describe the associated burst. In our numerical experiments, we have associated the burst with being the reconnection site. This identification is due to the similarity between the observed features and what is explained in the following. Further evidence of the relation between the reconnection site and bursts is found in the recent paper by Hansteen et al. (2017), where the authors show, through synthesis diagnostics of 3D simulations, that the bursts can be triggered in the mid and low chromosphere owing to the reconnection between different emerging bipolar magnetic fields.

In this paper, a good example of the burst is found at $x_{P}=14 \mathrm{Mm}$ for the slanted experiment (Figure 7) for $\theta=15^{\circ}$, which is an LOS parallel to the transition region at the reconnection site. Panel (A) shows that in that region (pink rectangle) there is a bidirectional flow with large upflow velocities, up to $85 \mathrm{~km} \mathrm{~s}^{-1}$, and downflow velocities of $<60$ $\mathrm{km} \mathrm{s}^{-1}$. In panel (D), we notice that the corresponding synthetic profile shares characteristics described in the observation for $\mathrm{R} 2 \mathrm{~b}$ (the brightest region of the Si IV burst): broad profiles with both red and blue components reaching large Doppler shifts. In the accompanying movie, we see that the blue component is usually brighter than the red one, which is also something found in the observation (see Section 3.2.2). Turning now to the vertical experiment, an example of the burst in a direction not parallel to the reconnection site can be found in the $\theta=15^{\circ}$ row of Figure 6 . Within the pink rectangle in panel (A), we see a downflow between $10.0 \leqslant x_{P} \leqslant 11.5 \mathrm{Mm}$, and a strong upflow around $11.5 \leqslant x_{P} \leqslant 13.0 \mathrm{Mm}$. The synthetic intensity shows large Doppler shifts for both components but with the blue component being brighter. In both experiments, we see that the plasma emitting in Si IV moves from the reconnection site to the detachment region where the internal footpoint of the surge is formed. This pattern of motion occurs repeatedly, a feature also found in the observation in $\mathrm{R} 2 \mathrm{~b}$ (see the second bullet point of Section 3.2.2). Finally, in the vertical experiment, there are also complex profiles due to the formation of plasmoids and subsequent cascade. The latter feature is addressed in more detail by Rouppe van der Voort et al. (2017) using the vertical numerical experiment and comparing it with plasmoids observed with CHROMIS at the SST.

\section{Discussion}

In this paper, we have used coordinated observations from IRIS and SST to study an $\mathrm{H} \alpha$ surge and accompanying Si IV burst episode occuring in active region AR 12585 on 2016 September 03. During the event, we found for the first time emission of Si IV within the surge and provide details about the properties of the surge and burst. In order to give a theoretical interpretation of the observations, we performed 2.5D RMHD numerical experiments of magnetic flux emergence through the 
granular cells right below the surface up to the atmosphere. The experiments were carried out with Bifrost using an extra module of the code that calculates the Si IV population in NEQ ionization. During the experiments, surges and bursts are obtained as a direct and indirect consequence of magnetic reconnection processes. We then compute synthetic spectra and compare with the observations.

In the following, we discuss some observational and theoretical features of the surge and burst and also some limitations of the present research.

\subsection{Observations}

Analyzing the light curves (Section 3.1), we have detected that in this case the surge and Si IV burst can be linked spatially and temporally to opposite polarity patches that converge and collide. Moreover, an EB is visible in close vicinity just prior to the start of the burst and remains active during the whole lifetime of the burst. It appears likely that all of these different phenomena share a common physical origin.

The most striking feature of the observation is the finding of emission in Si IV within the surge. Previous papers (e.g., Kim et al. 2015 and Huang et al. 2017) had only addressed the coexistence of surges, seen in $\mathrm{H} \alpha$ and $\mathrm{Ca}$ II $8542 \AA$, and Si IV brightenings, but without identifying evidences of Si IV within the surge. Our finding implies that surges, which are traditionally associated with chromospheric lines, have enough impact in the transition region to be detectable. Furthermore, this result together with the one by Madjarska et al. (2009), where the surge was associated with highly non-Gaussian line profiles in $\mathrm{OV}$ and $\mathrm{N} \mathrm{V}$, qualitatively increases the interest of studying transition region lines for diagnosis of chromospheric phenomena. The Si IV emission found in our surge is not strong, its intensity is around a factor of 2-5 brighter than a quiet-Sun typical Si IV profile, and sporadic: we detect it mostly in the rising phase of the surge, when it is visible in the blue wing of $\mathrm{H} \alpha$ (Section 3.2.1). The lack of Si IV in the decay phase of the surge may be due to cooling of the plasma so it is no longer emitting in Si IV or too weak to be detectable in short exposure time observations like the present one. Another interesting feature is that the brightest emission in Si IV within the domain of the surge is located near its footpoints. Moreover, the profiles are mainly redshifted with asymmetries to the blue. This reflects the complexity of the surge region, where in the same LOS we can find cool surge plasma visible in the blue wing of $\mathrm{H} \alpha$, which indicates rising motion, and hotter plasma emitting in Si IV, that is mainly descending, while there is a small fraction of large upflow velocities relative to the profile peak. The surge is otherwise of a canonical type: it is visible as an elongated and dark structure first in the blue and then in the red wings of $\mathrm{H} \alpha$ and $\mathrm{Ca}$ II, with projected lengths of several megameters, and Doppler velocities of a few tens of kilometers per second (Section 3.1). Concerning the Si IV burst (Section 3.2.2), we see typical burst characteristics as reported in the literature: the burst appears in regions on the surface where magnetic flux regions of opposite polarity converge and collide, it has broad profiles due to the presence of blue and red components, absorption features superimposed on the Si IV lines, brightenings in the wings of $\mathrm{Mg} \mathrm{II} \mathrm{h}$ and $\mathrm{k}$ lines with small enhancement in the cores, and enhanced wings in $\mathrm{H} \alpha$.

A full multi-diagnostic study of surges is challenging since it requires coordinated observations from different instruments.
Moreover, full spectral coverage requires the IRIS raster to coincide both spatially and temporally with the region where the events are occurring, which reduces the number of available observations for a statistical analysis. In our case, a severe limitation is the short exposure time of this observation $(0.5 \mathrm{~s})$, so lines like O IV 1401.2 and $1399.8 \AA$ cannot be used for density diagnostics since they require longer exposure times to be detectable (De Pontieu et al. 2014). Furthermore, the raster does not cover the whole surge, so we do not have a complete data set of the surge for a more complete comparison and questions about the emissivity in Si IV at the upper part of the surge remain open.

\subsection{Numerical Experiments}

In the paper, Si IV spectral synthesis has been used for the first time to study surges (Section 4.2.1) and the associated burst (Section 4.2.2). We have found that our models are able to reproduce some of the main features of the observations as summarized in the following.

1. The brightest points in Si IV within the surge correspond to the location around the footpoints of the surge. The excursions of the brightest patch from the observed surge footpoints are identified with the crests in the surge.

2. The synthetic Si IV profiles obtained in the experiment for the surge are mainly redshifted and the values fit with the observed Doppler shifts.

3. The less bright regions of the surge in Si IV seem to be brighter than an average transition region and match tentatively with what was found in the observations.

4. Our experiments provide a temporal and spatial relation between the surge and the burst. Both are a natural consequence of magnetic reconnection between emerged plasma and the pre-existing coronal magnetic field.

5. The motions observed with IRIS from the brightest region in the Si IV burst to the surge footpoints can be identified in the numerical experiments through plasma strongly emitting in Si IV that moves from the reconnection site to the internal footpoint of the surge.

6. The two-component profiles of the observed burst are characterized by large velocities and by the fact that the blue component is usually brighter than the red one: this is also found in the numerical experiments. The main difference between theory and observations concerns the burst intensity, which is substantially larger in the observations. A possible reason for the discrepancy is the lack of numerical resolution (Innes et al. 2015; Guo et al. 2017).

In the numerical experiments, we see that the orientation of the LOS plays an important role for the diagnosis. The resulting spectral intensity in the transition region lines can be enhanced by an important factor when the LOS runs parallel (or almost) to the local transition region. This is something to take into account for future interpretations from observations.

In spite of the good agreement with the observations, our current numerical experiments are not free from limitations, which are related to the challenging conditions in chromospheric plasma and associated radiative transfer. The experiments lack nonequilibrium ionization of hydrogen and helium, which could impact the properties of the emerged plasma and subsequent surge (Leenaarts et al. 2007; Golding et al. 2014). They also lack ambipolar diffusion, which has recently been shown to be key to obtaining related phenomena, like type II spicules (Martínez-Sykora et al. 2017). Moreover, our 
experiments are $2.5 \mathrm{D}$, but $3 \mathrm{D}$ modeling would be necessary to capture the full complexity of those phenomena, as was shown recently for bursts and EBs by Hansteen et al. (2017), and to be able to accurately synthesize the $\mathrm{H} \alpha$ line (Leenaarts et al. 2012). Proper $\mathrm{H} \alpha$ synthesis is important, e.g., to identify the numerical threads in the inner part of the surge (Section 4.1) with observed thread-like structures (Nelson \& Doyle 2013; Li et al. 2016), or to provide a theoretical explanation for the shocks associated with surges (Yang et al. 2014).

We gratefully acknowledge financial support from the Spanish Ministry of Economy and Competitiveness (MINECO) through projects AYA2011-24808 and AYA2014-55078-P, as well as from NASA contract NNG09FA40C (IRIS) and NASA grants NNH15ZDA001N-HSR and NNX16AG90G. IRIS is a NASA small explorer mission developed and operated by LMSAL with mission operations executed at NASA Ames Research center and major contributions to downlink communications funded by ESA and the Norwegian Space Centre. The Swedish 1-m Solar Telescope is operated on the island of $\mathrm{La}$ Palma by the Institute for Solar Physics (ISP) of Stockholm University in the Spanish Observatorio del Roque de los Muchachos of the Instituto de Astrofísica de Canarias. We also acknowledge the computer resources and assistance provided at the MareNostrum (BSC/CNS/RES, Spain) and TeideHPC (ITER, Spain) supercomputers. Finally, the authors are grateful to Gregal Vissers for his constructive comments during the Hinode-11/IRIS-8 science meeting.

\section{ORCID iDs}

D. Nóbrega-Siverio 주 https://orcid.org/0000-0002-7788-6482 J. Martínez-Sykora (i) https://orcid.org/0000-0002-0333-5717 L. Rouppe van der Voort (1) https://orcid.org/0000-00032088-028X

\section{References}

Asai, A., Ishii, T. T., \& Kurokawa, H. 2001, ApJL, 555, L65

Bong, S.-C., Cho, K.-S., \& Yurchyshyn, V. 2014, JKAS, 47, 311

Brooks, D. H., Kurokawa, H., \& Berger, T. E. 2007, ApJ, 656, 1197

Canfield, R. C., Reardon, K. P., Leka, K. D., et al. 1996, ApJ, 464, 1016

Cao, T.-j., Xu, A.-a., \& Tang, Y.-h. 1980, ChA, 4, 143

Carlsson, M., \& Leenaarts, J. 2012, A\&A, 539, A39

Chae, J., Qiu, J., Wang, H., \& Goode, P. R. 1999, ApJL, 513, L75

Chen, H. D., Jiang, Y. C., \& Ma, S. L. 2008, A\&A, 478, 907

de la Cruz Rodriguez, J. 2010, PhD thesis, Stockholm Univ.

de la Cruz Rodríguez, J., Löfdahl, M. G., Sütterlin, P., Hillberg, T., \& Rouppe van der Voort, L. 2015, A\&A, 573, A40

de la Cruz Rodríguez, J., Socas-Navarro, H., Carlsson, M., \& Leenaarts, J. 2012, A\&A, 543, A34

De Pontieu, B., McIntosh, S., Martinez-Sykora, J., Peter, H., \& Pereira, T. M. D. 2015, ApJL, 799, L12

De Pontieu, B., McIntosh, S. W., Hansteen, V. H., \& Schrijver, C. J. 2009, ApJL, 701, L1

De Pontieu, B., Title, A. M., Lemen, J. R., et al. 2014, SoPh, 289, 2733

Gaizauskas, V. 1996, SoPh, 169, 357

Golding, T. P., Carlsson, M., \& Leenaarts, J. 2014, ApJ, 784, 30

Grubecka, M., Schmieder, B., Berlicki, A., et al. 2016, A\&A, 593, A32

Gu, X. M., Lin, J., Li, K. J., et al. 1994, A\&A, 282, 240

Gudiksen, B. V., Carlsson, M., Hansteen, V. H., et al. 2011, A\&A, 531, A154 Guglielmino, S. L., Bellot Rubio, L. R., Zuccarello, F., et al. 2010, ApJ, 724, 1083 Guo, L.-J., De Pontieu, B., \& Peter, H. 2017, Natur, submitted Gupta, G. R., \& Tripathi, D. 2015, ApJ, 809, 82

Hansteen, V. H., Archontis, V., Pereira, T. M. D., et al. 2017, ApJ, 839, 22 Hayek, W., Asplund, M., Carlsson, M., et al. 2010, A\&A, 517, A49

Huang, Z., Madjarska, M. S., Scullion, E. M., et al. 2017, MNRAS, 464, 1753
Innes, D. E., Guo, L.-J., Huang, Y.-M., \& Bhattacharjee, A. 2015, ApJ, 813, 86 Jiang, Y. C., Chen, H. D., Li, K. J., Shen, Y. D., \& Yang, L. H. 2007, A\&A, 469,331

Jibben, P., \& Canfield, R. C. 2004, ApJ, 610, 1129

Judge, P. G. 2015, ApJ, 808, 116

Kim, Y.-H., Yurchyshyn, V., Bong, S.-C., et al. 2015, ApJ, 810, 38

Kirshner, R. P., \& Noyes, R. W. 1971, SoPh, 20, 428

Kurokawa, H., Liu, Y., Sano, S., \& Ishii, T. T. 2007, in ASP Conf. Ser. 369, New Solar Physics with Solar-B Mission, ed. K. Shibata, S. Nagata, \& T. Sakurai (San Francisco, CA: ASP), 347

Leenaarts, J., Carlsson, M., Hansteen, V., \& Rutten, R. J. 2007, A\&A, 473, 625

Leenaarts, J., Carlsson, M., \& Rouppe van der Voort, L. 2012, ApJ, 749, 136 Li, Z., Fang, C., Guo, Y., et al. 2016, ApJ, 826, 217

Liu, W., Berger, T. E., Title, A. M., \& Tarbell, T. D. 2009, ApJL, 707, L37

Liu, Y., \& Kurokawa, H. 2004, ApJ, 610, 1136

Madjarska, M. S., Doyle, J. G., \& de Pontieu, B. 2009, ApJ, 701, 253

Martínez-Sykora, J., De Pontieu, B., Hansteen, V., \& McIntosh, S. W. 2011, ApJ, 732, 84

Martínez-Sykora, J., De Pontieu, B., Hansteen, V. H., et al. 2017, Sci, 356, 1269

Martínez-Sykora, J., De Pontieu, B., Hansteen, V. H., \& Gudiksen, B. 2016, ApJ, 817, 46

Nelson, C. J., \& Doyle, J. G. 2013, A\&A, 560, A31

Nishizuka, N., Shimizu, M., Nakamura, T., et al. 2008, ApJL, 683, L83

Nóbrega-Siverio, D., Moreno-Insertis, F., \& Martínez-Sykora, J. 2016, ApJ, 822,18

Olluri, K., Gudiksen, B. V., \& Hansteen, V. H. 2013a, ApJ, 767, 43

Olluri, K., Gudiksen, B. V., \& Hansteen, V. H. 2013b, AJ, 145, 72

Olluri, K., Gudiksen, B. V., Hansteen, V. H., \& De Pontieu, B. 2015, ApJ, 802, 5

Ortiz, A., Bellot Rubio, L. R., Hansteen, V. H., de la Cruz Rodríguez, J., \& Rouppe van der Voort, L. 2014, ApJ, 781, 126

Pereira, T. M. D., Carlsson, M., De Pontieu, B., \& Hansteen, V. 2015, ApJ, 806, 14

Peter, H., Tian, H., Curdt, W., et al. 2014, Sci, 346, 1255726

Polito, V., Del Zanna, G., Dudík, J., et al. 2016, A\&A, 594, A64

Robustini, C., Leenaarts, J., de la Cruz Rodriguez, J., \& Rouppe van der Voort, L. 2016, A\&A, 590, A57

Rouppe van der Voort, L., De Pontieu, B., Pereira, T. M. D., Carlsson, M., \& Hansteen, V. 2015, ApJL, 799, L3

Rouppe van der Voort, L., De Pontieu, B., Scharmer, G. B., et al. 2017, ApJL, submitted

Roy, J.-R. 1973, SoPh, 32, 139

Rust, D. M. 1976, RSPTA, 281, 353

Rutten, R. J., Vissers, G. J. M., Rouppe van der Voort, L. H. M., Sütterlin, P., \& Vitas, N. 2013, Journal of Physics Conference Series, 440, 012007

Scharmer, G. B. 2006, A\&A, 447, 1111

Scharmer, G. B., Bjelksjo, K., Korhonen, T. K., Lindberg, B., \& Petterson, B. 2003, Proc. SPIE, 4853, 341

Schmahl, E. J. 1981, SoPh, 69, 135

Schmieder, B., Mein, P., Martres, M. J., \& Tandberg-Hanssen, E. 1984, SoPh, 94, 133

Schmieder, B., Shibata, K., van Driel-Gesztelyi, L., \& Freeland, S. 1995, SoPh, 156,245

Schmit, D. J., Innes, D., Ayres, T., et al. 2014, A\&A, 569, L7

Shimizu, T., Katsukawa, Y., Kubo, M., et al. 2009, ApJL, 696, L66

Tian, H., McIntosh, S. W., De Pontieu, B., et al. 2011, ApJ, 738, 18

Tian, H., Xu, Z., He, J., \& Madsen, C. 2016, ApJ, 824, 96

Uddin, W., Schmieder, B., Chandra, R., et al. 2012, ApJ, 752, 70

van Noort, M., Rouppe van der Voort, L., \& Löfdahl, M. G. 2005, SoPh, 228, 191

Vargas Domínguez, S., Kosovichev, A., \& Yurchyshyn, V. 2014, ApJ, 794, 140

Vissers, G. J. M., Rouppe van der Voort, L. H. M., \& Rutten, R. J. 2013, ApJ, 774,32

Vissers, G. J. M., Rouppe van der Voort, L. H. M., Rutten, R. J., Carlsson, M., \& De Pontieu, B. 2015, ApJ, 812, 11

Wang, J. f., Zhou, T. h., \& Ji, H. s. 2014, ChA\&A, 38, 65

Watanabe, H., Vissers, G., Kitai, R., Rouppe van der Voort, L., \& Rutten, R. J. 2011, ApJ, 736, 71

Yang, H., Chae, J., Lim, E.-K., et al. 2013, SoPh, 288, 39

Yang, H., Chae, J., Lim, E.-K., et al. 2014, ApJL, 790, L4

Zhang, J., Wang, J., \& Liu, Y. 2000, A\&A, 361, 759

Zhang, Q. M., \& Ji, H. S. 2014, A\&A, 561, A134

Zhelyazkov, I., Zaqarashvili, T. V., Chandra, R., Srivastava, A. K., \& Mishonov, T. 2015, AdSpR, 56, 2727 